\title{
Mining Features for Biomedical Data using Clustering Tree Ensembles
}

\author{
Konstantinos Pliakos, Celine Vens \\ KU Leuven, Campus KULAK, Department of Public Health and Primary Care, Faculty \\ of Medicine, 8500 Kortrijk, Belgium
}

\begin{abstract}
The volume of biomedical data available to the machine learning community grows very rapidly. A rational question is how informative these data really are or how discriminant the features describing the data instances are. Several biomedical datasets suffer from lack of variance in the instance representation, or even worse, contain instances with identical features and different class labels. Indisputably, this directly affects the performance of machine learning algorithms, as well as the ability to interpret their results. In this article, we emphasize on the aforementioned problem and propose a target-informed feature induction method based on tree ensemble learning. The method brings more variance into the data representation, thereby potentially increasing predictive performance of a learner applied to the induced features. The contribution of this article is two-fold. Firstly, a problem affecting the quality of biomedical data is highlighted, and secondly, a method to handle that problem is proposed. The efficiency of the presented approach is validated on multi-target prediction tasks. The obtained results indicate that the proposed approach is able to boost the discrimination between the data instances and increase the predictive performance.
\end{abstract}


Keywords: Tree-ensembles, Extremely randomized trees, Tree-embeddings, Biomedical data mining

\section{Introduction}

Significant progress has been made in biomedical data generation techniques and feature extraction methods. Microarrays, high-throughput sequencing, mass spectrometry and many others have been recognized as tools

5 of great importance. The scientific community exploits these technological advances to generate more data in the field of biomedicine. However, a thoughtful question would be how informative these data truly are to make predictive inferences.

More precisely, among all the scientific tools in biomedical informatics, machine learning has proved to be of paramount importance, especially in predictive problems. It has provided contributions in a wide range of problems in biology and biomedicine [1, 2]. Protein function prediction, interaction prediction between drugs and proteins, knowledge discovery related to biomarkers are only some of the various examples. In particular, in the framework of supervised learning several methods are provided to learn predictive models only from former observations of a system. In supervised learning, the instances are described by features (characteristics of each instance) and accompanied by targets. The goal is to learn a model on a training set of instances that can predict the target given the features 3 . This model is then used to predict the target of new unseen instances. If the target is numeric, the task is called regression. If the target is categorical (i.e., a class has to be predicted), the term classification is used. In some 
prediction problems, rather than a single target, a set of targets needs to be predicted. The mentioned applications of gene function prediction or interaction prediction are instances of this so-called multi-target prediction task [4. Multi-target prediction is a generalization of multi-target regression and multi-target classification. Multi-label classification can be considered as an instance of multi-target classification with only two nominal values for each target [5, 6]. These applications can be tackled by either transforming the multi-target problem into a set of single-target problems and applying a standard prediction algorithm, or by applying a specific multi-target prediction algorithm.

One of the most popular machine learning algorithms are the decision tree induction algorithms [7]. They have been applied extensively in biological and biomedical systems [2, 8]. Decision tree algorithms have many advantages over other machine learning methods. They provide interpretability of the induced models, providing transparency and insights to scientists, leveraging this way knowledge discovery. Their models are also scalable, computationally efficient and accurate. The predictive performance of decision trees is specifically boosted when they are combined with ensemble methods [9], providing state-of-the-art results. Decision tree learning and its ensemble extension have been extended to the multi-target prediction setting (i.e., multi-label classification and multi-target regression) [4]. Multi-label classification has received more attention than multi-target regression [10].

\subsection{Problem Statement}

In biomedicine and healthcare representing the data effectively is often a challenging task. In comparison to other fields, such as multimedia anal- 
ysis or web mining, getting new features for an instance is a substantially more difficult task in biomedicine. In the case of a song or an image being under-represented one can easily get (extract) more features by collecting more information about the artist or applying an audio or image processing technique to the instance itself. However, this is not always possible in biomedicine. It requires significant time, expenditure, and the presence of human experts, as the features most of the times are the outcome of laboratory work. Furthermore, in medicine the data are usually produced through the process of patient care and thereby issues of privacy are included [1]. In many countries, hospitals are equipped with inefficient database systems that are mainly designed for billing purposes. In addition, medical data inevitably contain many missing values and many non-numeric features. Many traditional machine learning methods (e.g., SVM [11]) can not be applied on such data without preprocessing. These peculiarities of the data in healthcare and biomedicine often lead to non-variant data representations and inevitably the harassment of the performance of machine learning methods [12]. To this end, methodologies to handle this uniqueness of biomedical data are needed.

It is crucial for the performance of machine learning models that the features that describe the data instances are enriched with valuable information and are able to discriminate the data instances. However, there are many examples of broadly used benchmark biomedical datasets suffering from this phenomenon of lacking variance. An extreme version of the aforementioned 70 phenomenon is the existence of biomedical datasets that contain identical feature vectors for different data instances. Some examples from the field of gene function prediction (e.g., [13, [14]) or interaction prediction (e.g., 
[15]) are shown in Table 11 [16, 17]. This is irrational as there are instances (e.g., genes), that have different targets (e.g., functions) but identical feature vectors.

Table 1: Datasets, the number of features, instances and unique feature vectors.

\begin{tabular}{ccccc}
\hline Context & Dataset & $\mid$ features $\mid$ & |instances $\mid$ & $\begin{array}{c}\mid \text { unique feature } \\
\text { vectors } \mid\end{array}$ \\
\hline Gene fct. prediction & church & 27 & 3755 & $\mathbf{2 3 5 2}$ \\
(S. cerevisiae) & pheno & 69 & 1592 & $\mathbf{5 1 4}$ \\
& hom & 47034 & 3854 & 3646 \\
& seq & 478 & 3919 & 3913 \\
& struc & 19628 & 3838 & 3785 \\
\hline (A. thaliana) & scop & 2003 & 9843 & 9415 \\
& struc & 19628 & 11763 & 11689 \\
\hline Interaction prediction & drugs & 660 & 1862 & 1779 \\
(DPI network) & proteins & 876 & 1554 & $\mathbf{6 8 3}$ \\
\hline
\end{tabular}

In the pheno dataset for example, $32 \%$ of the instances have a unique feature vector. As a unique feature vector we denote a feature vector that exists only once in the dataset after removing the replicates (i.e., instances that are described by exactly the same feature vectors). This dataset contains an instance for every gene in the $S$. cerevisiae organism, and the instance corresponds to a mutant, i.e. an organism where the corresponding gene has been altered. There are 69 features corresponding to different growth medium (e.g., caffeine, sorbitol, benomyl,...), on which growth of the mutant is recorded. The values of the features correspond to the observed sensitivity 
or resistance of the mutant: no effect, less growth or better growth compared to the wild type, or no data for the growth medium. The large number of replicates (i.e., instances that are described by exactly the same feature vectors) can be explained by two observations: (1) the data is very sparse, i.e. for many mutants few growth media have been tested, and (2) in many cases the tested growth media has no effect on the mutant. The church dataset also contains many instances with identical feature values. It consists of 27 mostly real-valued features. Interestingly, the replicate issue is not limited to datasets with few features. For example, hom contains homology information encoded by 47034 binary features, struc contains 19628 binary features related to predicted secondary structure of the protein. Moreover, apart from genes the aforementioned problem is also existing in interaction networks. In Table 1, an example of a drug-protein interaction network (DPI) [18] is presented where there are some drugs or proteins having the same feature vector. In drug-protein interaction networks connections are formed between drugs and proteins when the drug targets the protein. Both interaction parts are described by their own set of features, for example in the current dataset each drug is described by the presence or absence of 660 chemical substances and each protein by the presence or absence of 876 PFAM domains.

Lack of variance in the data representation can heavily impair the performance of machine learning algorithms. Indicatively, by applying an 1 nearest neighbor (1-NN) classifier to a training dataset (including the specific query instance), one expects to get $100 \%$ accuracy. However, in case there are instances in that dataset with exactly the same feature representation, it is not 
Whereas numerous studies have focused on feature selection [20, 21, 22], the more difficult task of feature construction or induction has received less attention. The main goal of feature construction is to augment the feature space by creating or inferring additional features [23]. 

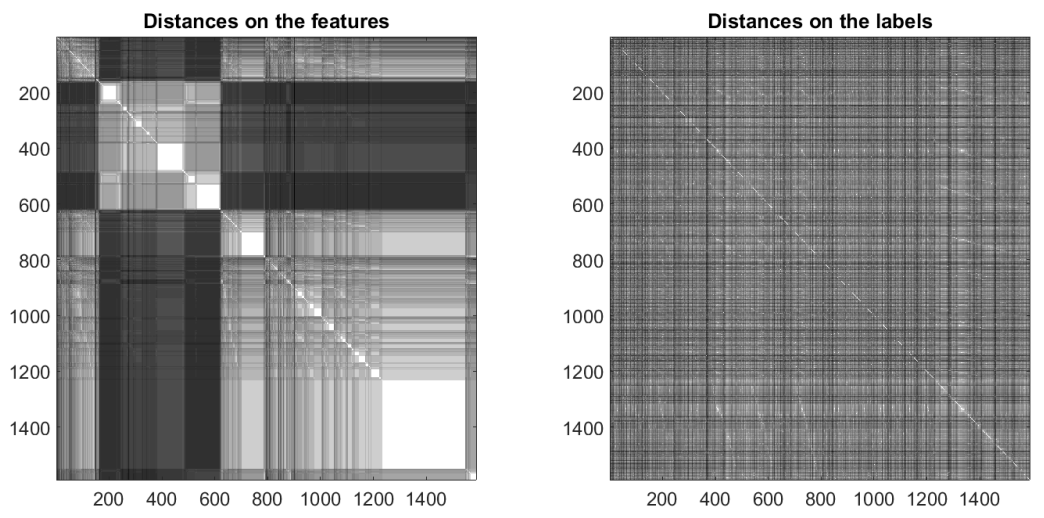

Figure 1: Distance matrices for the features (left) and the labels (right) of the pheno dataset.

In [24], a feature construction method specifically focused on multi-label classification was presented. A distinct feature set was assigned to every label, increasing this way the performance of a classifier trained for that specific label. The label-specific features were generated by first clustering the positive and negative instances (separately) of the label. Next, the distances of each instance to the obtained cluster centroids were calculated. Vens and Costa [25] proposed a feature induction technique based on random forest [9]. A metric transformation was proposed, mapping the identity of the tests performed in each node of a decision tree to a feature indicator. Next, the final representation is yielded by concatenating the features associated with the trees in the forest and encoding them with hashing. The method was demonstrated on binary and multi-label classification tasks. A similar work involving a set of random clustering forests was proposed in [26, 27]. The application was focused on the construction of visual vocabularies. More specifically, randomized trees were used to generate the new feature repre- 
sentation. The feature generation process took into account the indices of the leaves of those trees. The proposed method generated a high dimensional, sparse, and binary feature set. In [28, 29], an unsupervised feature induction technique based on Extremely Randomized Trees [30] was presented. The feature representation was also extended towards interaction data in [29] and 31. Moreover, there have been many studies that adopted methods from the field of dimensionality reduction [32] for feature extraction. In [33], a comparison study between Principal Component Analysis (PCA), Independent Component Analysis (ICA), and Kernel Principal Component Analysis (KPCA) was performed. Furthermore, in [34], deep auto-encoder networks were used for dimensionality reduction, extracting features from high dimensional vectors. More specifically, an efficient approach was proposed to initialize the weights of these multi-layer neural networks, assisting auto-encoders to learn low-dimensional representations. Here, we focus on feature construction for multi-target prediction problems using scalable and inductive models that provide also interpretability (e.g., feature ranking).

\subsection{Introduction to the proposed method}

Motivated by the issue of lacking variance in biomedical data that was presented above we propose an efficient feature induction method that transforms the data into more variant and more informative representations. More specifically, we propose a method motivated by [25, 28]. The proposed approach yields a feature set using Extremely Randomized Trees [30]. In particular, the nodes of each tree of the forest are considered clusters that comprise the instances that pass through them. In the next step, binary vectors are produced. The existence of an instance in a cluster-node is associated with 

background of our approach and presenting the ensemble method where our approach is based on. Next, we present the feature induction approach in 
detail. and the overfitting effect. These disadvantages are surpassed by extending the PCT framework to ensembles of trees. Various tree ensemble models exist. Here, we consider the ensemble method of Extremely Randomized Trees 
(ERT) [30. In an ERT ensemble, each tree is constructed by considering

\subsection{Feature induction with ERT}

The proposed approach starts by applying Extremely randomized trees to the original feature set. Let $\mathbf{N}=\left\{n_{1}, n_{2}, \cdots, n_{|N|}\right\}$ be the nodes of all trees in the ensemble. The tree-nodes are treated as clusters. The instances contained 


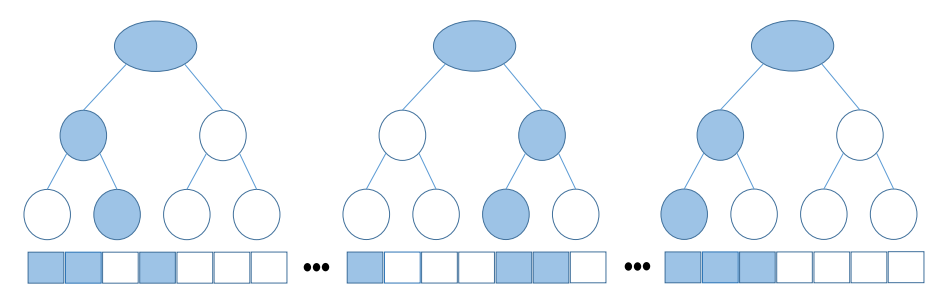

Figure 2: Illustration of the proposed approach. An instance traverses the trees and the corresponding feature vector is constructed.

in those clusters are the ones that pass through them while traversing the tree. 250 The original feature set is denoted as $\mathbf{X} \in \Re^{|S| \times|M|}$ and the generated one as $\mathbf{F} \in \Re^{|S| \times|N|}$, where $|S|$ and $|N|$ correspond to the number of instances and the number of the generated features of the dataset, respectively. Although the trees of the ensemble may contain different number of nodes, all the instances traverse all the trees and therefore the corresponding generated 255 feature vectors are of equal size (i.e., the total number of nodes $|N|$ ). The clusters $n_{j} \in \mathbf{N}$ are treated as features of the feature set $\mathbf{F}$. Each $f_{i j} \in \mathbf{F}$ equals to 1 if the instance $i \in \mathbf{S}$ is contained in cluster (node) $j \in \mathbf{N}$ and 0 otherwise, as shown in equation (11). When it comes to new (unseen) instances, they also traverse the trees of the ensemble, generating feature vectors. In Fig. 2, the tree-ensembles part of the feature induction approach is illustrated.

$$
f_{i j}= \begin{cases}1, & \text { if } i \in n_{j} \\ 0, & \text { otherwise }\end{cases}
$$

In order to generate a better feature representation the features corresponding to the cluster nodes of every tree are weighed and filtered based on the number of instances they contain (i.e., weight of the cluster). In order to preserve the inductive setup of the proposed method these weights are 
computed only once (i.e., in the training phase) and they are not subject to new instances (i.e., they are not updated when new instances arrive). The weight of every node $j$ is computed as in equation 2 .

$$
w_{j}=\frac{1}{\log \left(\left|n_{j}\right|\right)+e}
$$

Where $\left|n_{j}\right|$ is the number of the instances contained in the cluster (node) $270 j$ and $e$ a very small value $(e \ll 1)$ in order to guarantee the numerical stability of the fraction (i.e., the denominator of the fraction is never equal to 0 ). The features that correspond to the nodes close to the root are not so informative as they often contain approximately all the instances of a dataset. By discarding the nodes containing more than $p \%$ of the instances, the number of generated features is reduced making the subsequent learning phase more efficient. Based on this strategy, one can keep all the nodes from root to leaves, discard the root nodes and some nodes close to them, or keep only the leaves. Next, the data are transfered from a high dimensional space to a low dimensional space through maximizing the variance in the data representation. This is performed by applying PCA. The high dimensional feature vectors are converted to a low dimensional orthogonal basis set. The fitted model can then be also used for new instances as well. Note that we also suggest the removal of many components. By keeping only few of the first components, which are the most informative (most variant), we achieve noise removal and make the generated feature set more computationally efficient for subsequent machine learning analysis. Although the new features cannot be interpreted, the ERT employed in the first phase of the method provides a feature ranking output that could help scientists that want to get some insights from their features. This is the same level of interpretability one gets 

re-training the model). 
Table 2: The datasets used in the evaluation procedure are presented.

\begin{tabular}{|l|c|c|c|c|}
\hline \multicolumn{1}{|c|}{ Dataset } & $\mid$ instances $\mid$ & $\mid$ Features $\mid$ & $\mid$ targets $\mid$ & Type of Targets \\
\hline ERN & 1164 & 445 & 154 & Binary \\
SRN & 1821 & 9884 & 113 & Binary \\
MN & 668 & 325 & 668 & Binary \\
PPI & 984 & 325 & 984 & Binary \\
Medical & 978 & 1449 & 45 & Binary \\
Yeast & 2417 & 103 & 14 & Binary \\
\hline WQ & 1060 & 16 & 14 & Numeric \\
SG & 735 & 113 & 18 & Numeric \\
\hline
\end{tabular}

\subsection{Datasets}

In our study, we used in total 8 datasets from the fields of biology and

- E. coli regulatory network (ERN) [36]. This dataset corresponds to a regulatory network. It consists of 1164 genes of E. coli. Each gene is represented by 445 expression values (i.e., these are the features). The binary targets represent the interactions between these genes and 154 Transcription Factors (TFs). 
- S. cerevisiae regulatory network (SRN) [37]. This dataset corresponds to a regulatory network. It consists of 1821 genes of S. cerevisiae regulated by 113 Transcription Factors (TFs). Each gene is represented by 1685 expression values and 8199 motifs features. The binary targets represent the connections between the Transcription Factors (TFs) and their target genes.

- Metabolic Network (MN) [38] This dataset represents an homogeneous metabolic network. The network is composed of $668 \mathrm{~S}$. cerivisiae enzymes. The 668 targets have binary values and they represent the edges of the network. There is an edge (i.e, target value is equal to 1) between two enzymes when these two enzymes catalyse successive reactions. Each enzyme is described by 325 features (a concatenation of expression data, phylogenetic profiles, and localization data).

- Protein-Protein Interaction Network (PPI) [39] This dataset represents an homogeneous protein-protein interaction network. The network is composed of $984 \mathrm{~S}$. cerivisiae proteins. The 984 targets have binary values and they represent the edges of the network. There is an edge (i.e, target value is equal to 1) between two proteins when these two proteins interact with each other. Each protein is described by 325 features (a concatenation of expression data, phylogenetic profiles, and localization data).

- Yeast [40] This dataset contains information about a set of 2417 Yeast cells described by 103 features. The task is to determine the localization site of each cell. The dataset contains 14 binary targets. 
- Medical 41] This dataset contains 978 instances of clinical text docwere the area under precision recall curve (AUPR) and the area under the receiver operating characteristic curve (AUROC). A PR curve is defined as the Precision $\left(\frac{T P}{T P+F P}\right)$ against the Recall $\left(\frac{T P}{T P+F N}\right)$ at various thresholds. A ROC curve plots the true positive rate $\left(\frac{T P}{T P+F N}\right)$ on the $y$ axis against the false 370 positive rate $\left(\frac{F P}{F P+T N}\right)$ on the $x$ axis at various thresholds. The true-positive rate is the same as recall, and is also known as sensitivity while the false 
positive rate is also known as (1-specificity). The aforementioned measures were employed in a micro-average setup. For multi-target regression, the Relative Root Mean Squared Error (RRMSE) was used as in equation (3).

$$
\operatorname{RRMSE}\left(y_{i}, \hat{y}_{i}\right)=\sqrt{\frac{\sum_{1}^{T}\left(y_{i}-\hat{y}_{i}\right)^{2}}{\sum_{1}^{T}\left(\bar{Y}_{i}-\hat{y}_{i}\right)^{2}}}
$$

375

Where $\hat{y}_{i}$ is the predicted value of the $i$-th instance, $y_{i}$ is the corresponding true value, $\bar{Y}_{i}$ is the mean value of the target variable over the training set, and $T$ is the number of the test instances. The measure was applied in a uniform averaging setup (i.e., the errors of all outputs were averaged with a uniform weight). For evaluation purposes, the 5 -fold cross validation setting was followed. The proposed approach is denoted as Low dimensional Clustering Tree Features $(L C T F)$ and was compared to the original feature representation $(O R F)$, Kernel PCA features $(K P C A F)$, and Random Forest feature induction $(R F F I)$ [25]. For the training of the tree ensembles (LCTF), the number of trees was set equal to 200. As stopping criterion, the minimum number of instances that have to be present in a leaf was used and it was set equal to 5 . These parameters do not affect significantly the training procedure (e.g., one could use 100 or 300 trees and 3 or 8 as a stopping criterion without observing major differences in the results). Moreover, the tree node weight scheme was applied as described in Section 2.2 and the nodes containing more than $90 \%$ of all the data instances were removed (i.e., mainly the root nodes and some nodes close to them). For KPCA, the radial basis function $(\mathrm{RBF})$ kernel (i.e., $\left.K(\chi, \psi)=-\gamma\|\chi-\psi\|^{2}\right)$ was employed and the $\gamma$ parameter was set equal to $1 /|M|$, where $M$ is the number of the original features. For RFFI, we used 200 trees (in the original paper [25] the algo- 
rithm converged at this number) and a stopping criterion of 5 instances per leaf (the same as in our algorithm). In order to evaluate the efficiency of the generated feature representation in multi-label classification, the ML-KNN (Multi-Label K-Nearest Neighbor) [19] algorithm was employed. Multiple numbers of nearest neighbors were used and the smoothing parameter was set equal to 1 (i.e., Laplacian smoothing, as in the original paper [19]). For multi-target regression, we used the k-NN regressor (i.e., for each test, the target vector is predicted by averaging the target vectors associated with the nearest neighbors in the training set). Multiple numbers of nearest neighbors were used. The computation of the nearest neighbors was based on Euclidean distances. As we are interested in evaluating the contribution of the features we selected a relatively simple predictor. For the implementation and experimental evaluation we used the machine learning library Scikit-learn (Python) [44, and the libraries NumPy and matplotlib [45].

\subsection{Experimental Results and Discussion}

In Fig. 3, the 2D (two dimensional) data distribution of 4 datasets is displayed (see supplementary files for all the 2D and 3D projections). The original feature sets (ORF) were projected to 2D using PCA. Using the original features, it is noted that no manifold is detected and the data projections are similar to a random projection. This is particularly obvious in cases of wq and ern datasets. As reflected, the LCTF generates better data representations than original features. More specifically, it is shown that using LCTF adds variance between the instances and improves the data representation in cases where the original features can not sufficiently discriminate the instances. 

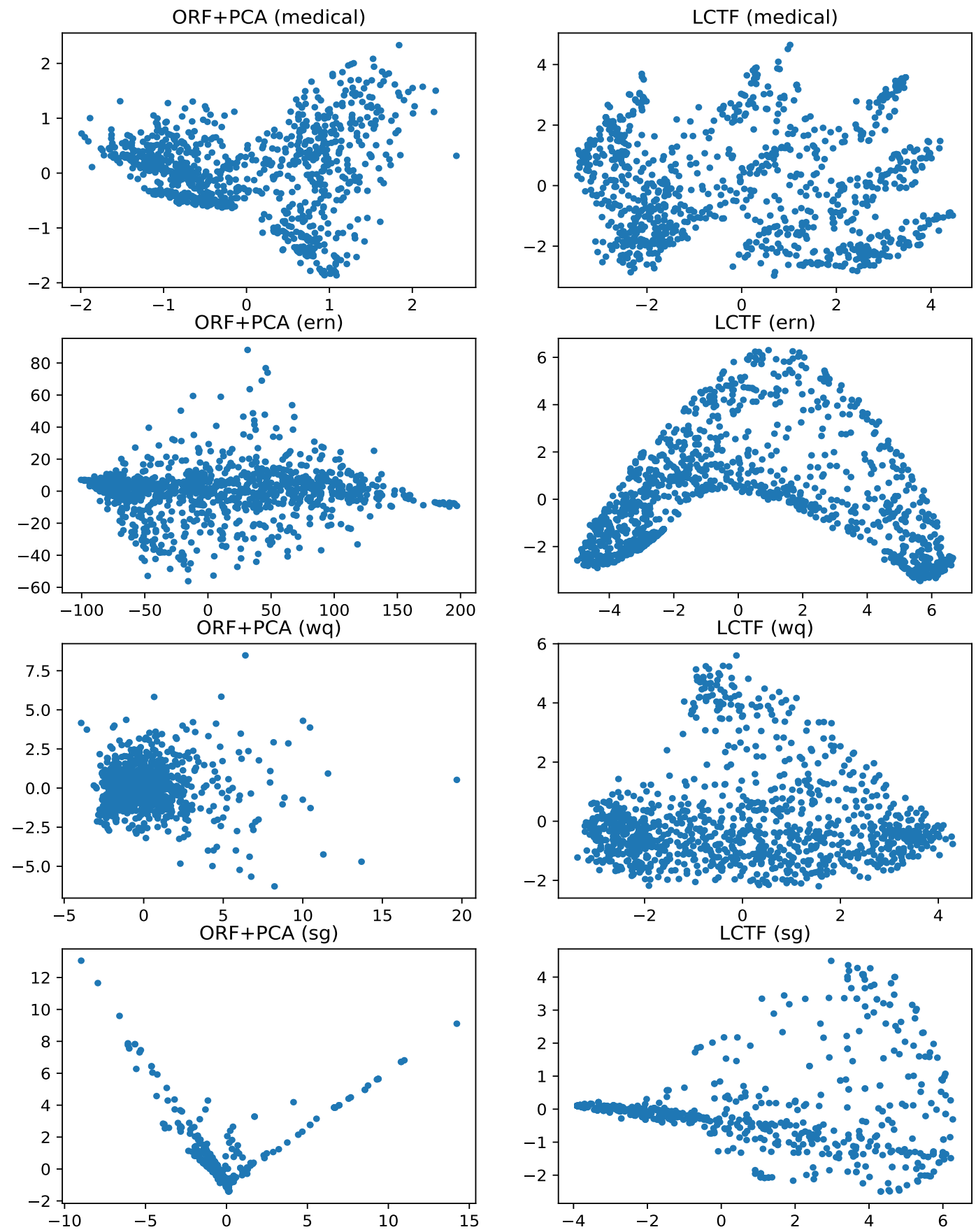

Figure 3: 2D projections of 4 datasets used in our evaluation procedure. 

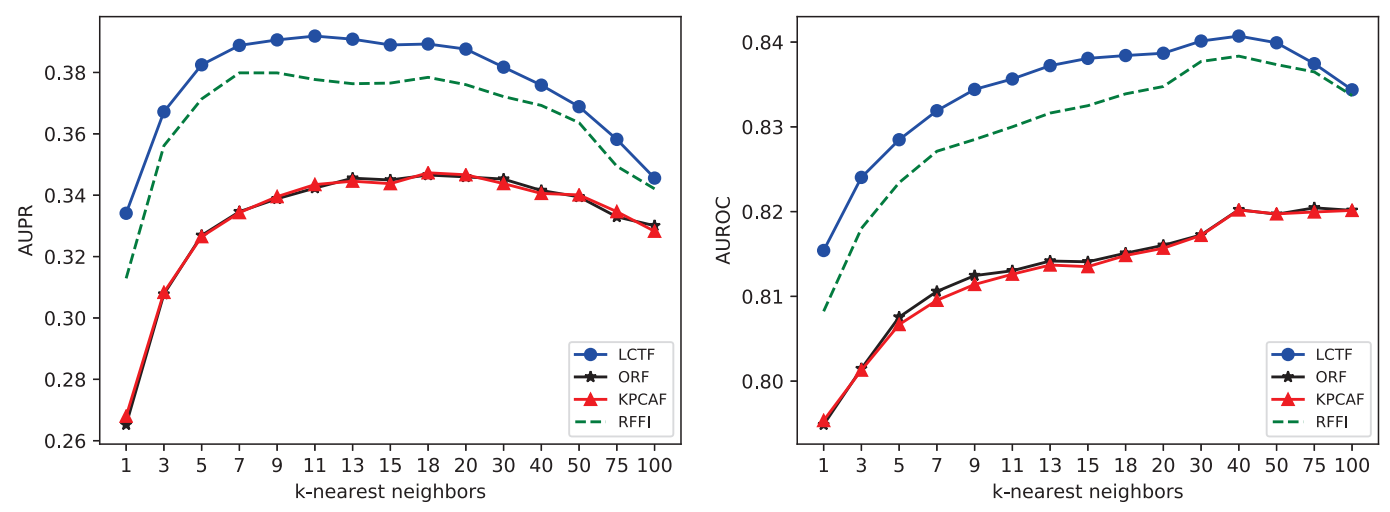

Figure 4: Average AUROC and AUPR results for various numbers of nearest neighbors.

In Table 3, the obtained comparison results for multi-label classification are illustrated. We compared our generated feature set to the original features, to the feature representation yielded by KPCA and to the Random Forest induced feature representation. Standard deviations are also provided in parenthesis and the best results are indicated in bold. For ML-KNN, 9 nearest neighbors $(k=9)$ were used. As shown in Table 3 , the proposed approach outperforms the compared ones in terms of both AUROC and AUPR. Only in two cases (i.e., ERN, SRN datasets) LCTF is slightly outperformed by other approaches. More specifically, in the SRN dataset, the RFFI achieves better results and in the ERN, the ORF and KPCAF slightly surpass the proposed LCTF.

In Fig. 4, the average AUPR and AUROC results for all datasets are shown for various numbers of nearest neighbors. The results obtained by LCTF surpass the ones of the compared approaches. The obtained results for multi-target regression are illustrated in Table 4. It is shown that LCTF 435 is better than all the compared approaches. The number of nearest neigh- 
Table 3: AUPR and AUROC results for the compared approaches.

\begin{tabular}{|c|c|c|c|c|}
\hline \multicolumn{5}{|c|}{ AUPR } \\
\hline Data & LCTF & ORF & KPCAF & RFFI \\
\hline MN & $\mathbf{0 . 2 1 4}( \pm 0.05)$ & $0.095( \pm 0.01)$ & $0.095( \pm 0.01)$ & $0.147( \pm 0.026)$ \\
PPI & $\mathbf{0 . 1 5 5}( \pm 0.042)$ & $0.131( \pm 0.042)$ & $0.131( \pm 0.042)$ & $0.148( \pm 0.034)$ \\
ERN & $0.356( \pm 0.04)$ & $\mathbf{0 . 3 6 3 ( \pm 0 . 0 2 9 )}$ & $\mathbf{0 . 3 6 3}( \pm 0.029)$ & $0.354( \pm 0.036)$ \\
SRN & $0.13( \pm 0.014)$ & $0.047( \pm 0.005)$ & $0.045( \pm 0.002)$ & $\mathbf{0 . 1 6 1}( \pm 0.012)$ \\
Medical & $\mathbf{0 . 7 8 6}( \pm 0.051)$ & $0.697( \pm 0.046)$ & $0.703( \pm 0.039)$ & $0.772( \pm 0.051)$ \\
Yeast & $\mathbf{0 . 7 0 3 ( \pm 0 . 0 0 9 )}$ & $0.699( \pm 0.005)$ & $0.699( \pm 0.005)$ & $0.697( \pm 0.01)$ \\
\hline Average & $\mathbf{0 . 3 9 1}( \pm 0.034)$ & $0.339( \pm 0.023)$ & $0.339( \pm 0.021)$ & $0.38( \pm 0.028)$ \\
\hline & & AUROC & & \\
\hline Data & LCTF & ORF & KPCAF & RFFI \\
\hline MN & $\mathbf{0 . 8 1 8 ( \pm 0 . 0 2 3 )}$ & $0.735( \pm 0.015)$ & $0.735( \pm 0.015)$ & $0.782( \pm 0.025)$ \\
PPI & $\mathbf{0 . 7 8 9}( \pm 0.022)$ & $0.774( \pm 0.028)$ & $0.774( \pm 0.028)$ & $0.778( \pm 0.022)$ \\
ERN & $\mathbf{0 . 8 2}( \pm 0.03)$ & $\mathbf{0 . 8 2}( \pm 0.031)$ & $\mathbf{0 . 8 2}( \pm 0.031)$ & $0.819( \pm 0.028)$ \\
SRN & $0.792( \pm 0.012)$ & $0.766( \pm 0.006)$ & $0.761( \pm 0.005)$ & $\mathbf{0 . 8 1 1}( \pm 0.009)$ \\
Medical & $\mathbf{0 . 9 5 2 ( \pm 0 . 0 1 3 )}$ & $0.948( \pm 0.01)$ & $0.947( \pm 0.012)$ & $0.951( \pm 0.016)$ \\
Yeast & $\mathbf{0 . 8 3 6}( \pm 0.004)$ & $0.832( \pm 0.004)$ & $0.832( \pm 0.004)$ & $0.831( \pm 0.005)$ \\
\hline Average & $\mathbf{0 . 8 3 5}( \pm 0.017)$ & $0.813( \pm 0.016)$ & $0.812( \pm 0.016)$ & $0.829( \pm 0.018)$ \\
\hline
\end{tabular}


Table 4: RRMSE results for the compared approaches.

\begin{tabular}{|c|c|c|c|c|}
\hline \multicolumn{5}{|c|}{ RRMSE } \\
\hline Data & LCTF & ORF & KPCAF & RFFI \\
\hline WQ & $\mathbf{0 . 9 8 8}( \pm 0.034)$ & $0.997( \pm 0.022)$ & $0.997( \pm 0.022)$ & $0.996( \pm 0.036)$ \\
SG & $\mathbf{0 . 9 3 8 ( \pm 0 . 0 1 8 )}$ & $0.96( \pm 0.013)$ & $0.958( \pm 0.012)$ & $0.949( \pm 0.007)$ \\
\hline Average & $\mathbf{0 . 9 6 3}( \pm 0.026)$ & $0.9785( \pm 0.018)$ & $0.978( \pm 0.017)$ & $0.973( \pm 0.022)$ \\
\hline
\end{tabular}

bors was set equal to 9 . The average results for various numbers of nearest neighbors are illustrated in Fig. 5 .

It has to be mentioned that for evaluation purposes in the aforementioned experiments no PCA or KPCA generated components were removed. However, as stated in Section 2.2, the removal of many components is suggested. We conducted experiments removing each time a different percentage of components (i.e., amount of information). We compared the obtained results of the proposed approach (LCTF) to KPCAF, Independent Component Analysis (ICA) [46], Truncated SVD (similar to PCA), Locally Linear Embedding (LLE) [47, and Isomap [48] [32]. The results are shown in Figs. 66 and 7, for multi-label classification (ML-KNN, $k=9$ ) and multi-target regression (K$\mathrm{NN}, k=9$ ), respectively. LCTF outperforms all the compared approaches. As it is shown the performance of LCTF is not decreased by the reduction of the components. It is interesting to notice that the best performance is achieved when $80 \%-90 \%$ of the components are removed. This way, it can be deducted that the proposed method succeeds in generating a compact low 


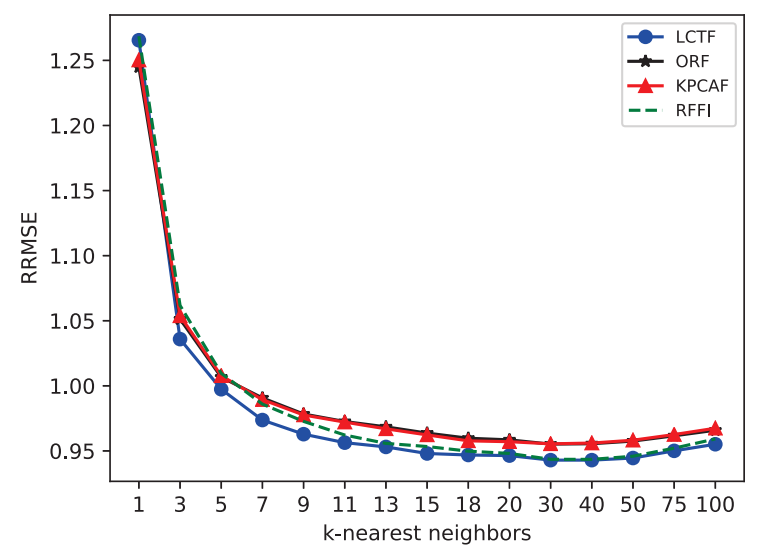

Figure 5: Average RRMSE results for various numbers of nearest neighbors.

dimensional feature representation, enriching the information contained in the data, inducing variance between the instances and removing the existing noise in the original representation.

In our evaluation process we computed the AUPR and AUROC in a micro-average setup. Nevertheless, one could argue that a macro-average setup is more suitable when it comes to class imbalanced datasets, as microaverage calculates a global measure using the total number of TP, TN, FP, FN and therefore can be biased towards the majority class. In Fig. 8, macroaveraged AUPR and AUROC results for multiple numbers of nearest neighbors are illustrated for the Yeast dataset. However, the computation of the macro-averaged measures presents a problem in cases of extreme class imbalance, where there are targets containing very few (sometimes only one) positive labels. In such cases, multiple folds may be left without any positive labels for some targets, leading to an undefined recall. This case is present in the rest of the multi-label datasets used here (i.e., MN, PPI, ERN, SRN, 

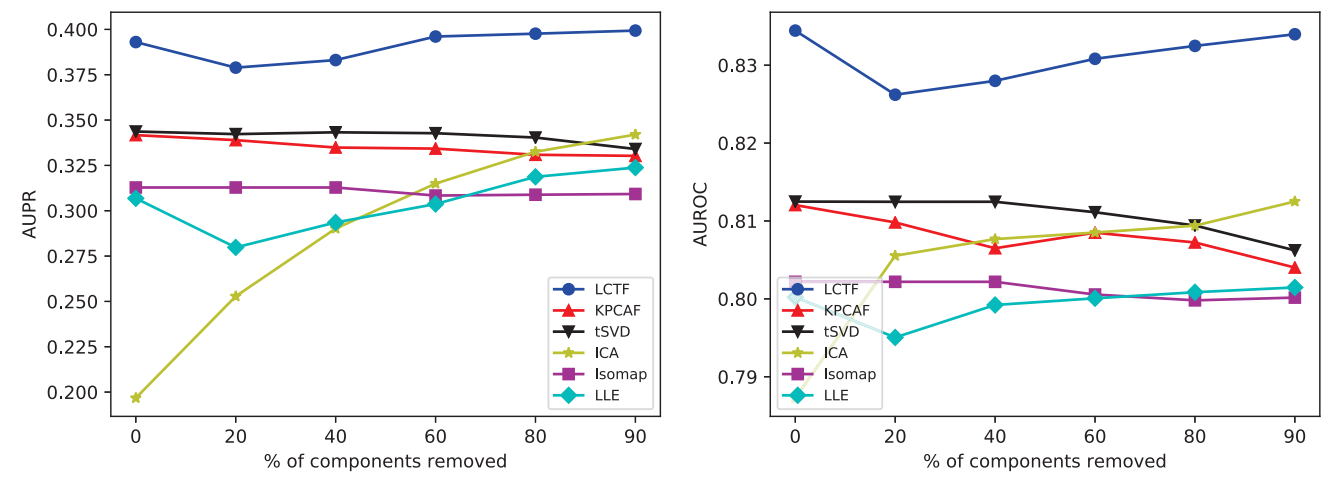

Figure 6: Average AUROC and AUPR results for various percentages of removed components.

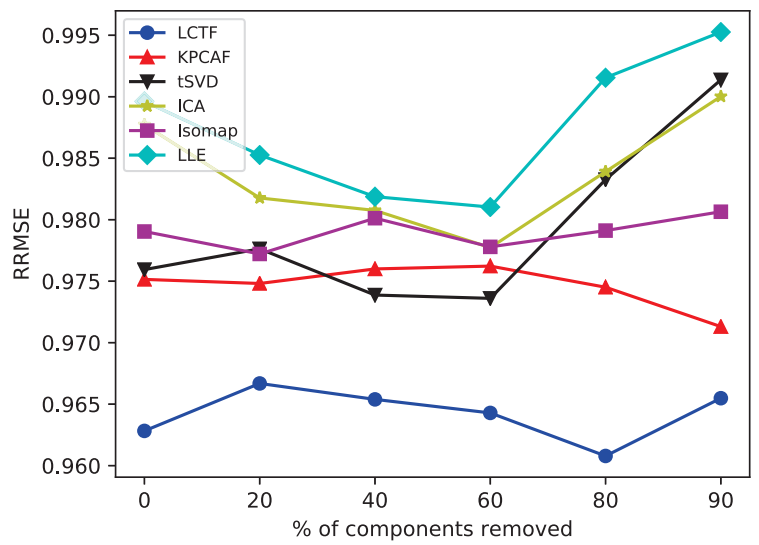

Figure 7: Average RRMSE results for various percentages of removed components. 

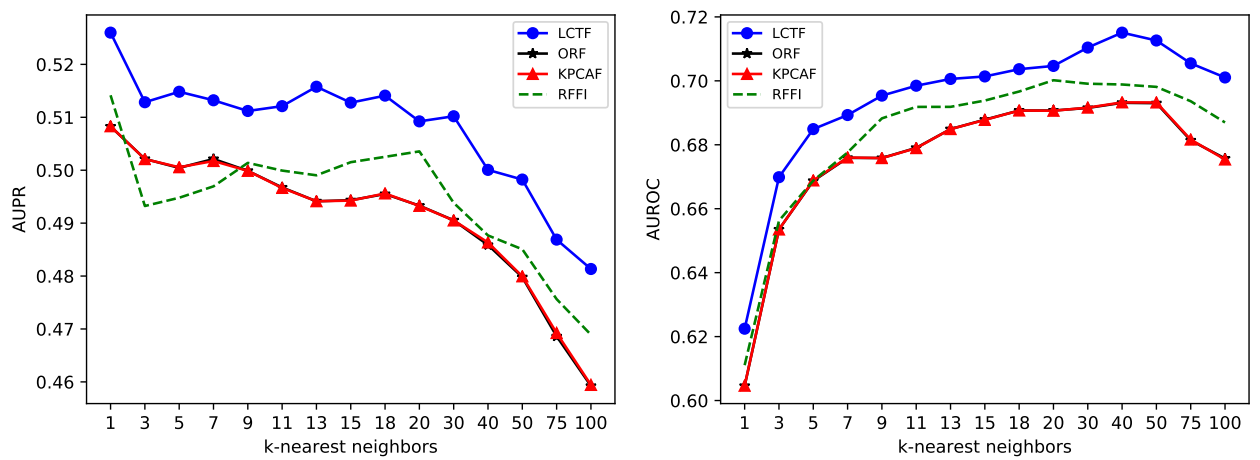

Figure 8: Macro-averaged AUPR and AUROC results for various numbers of nearest neighbors (Yeast dataset).
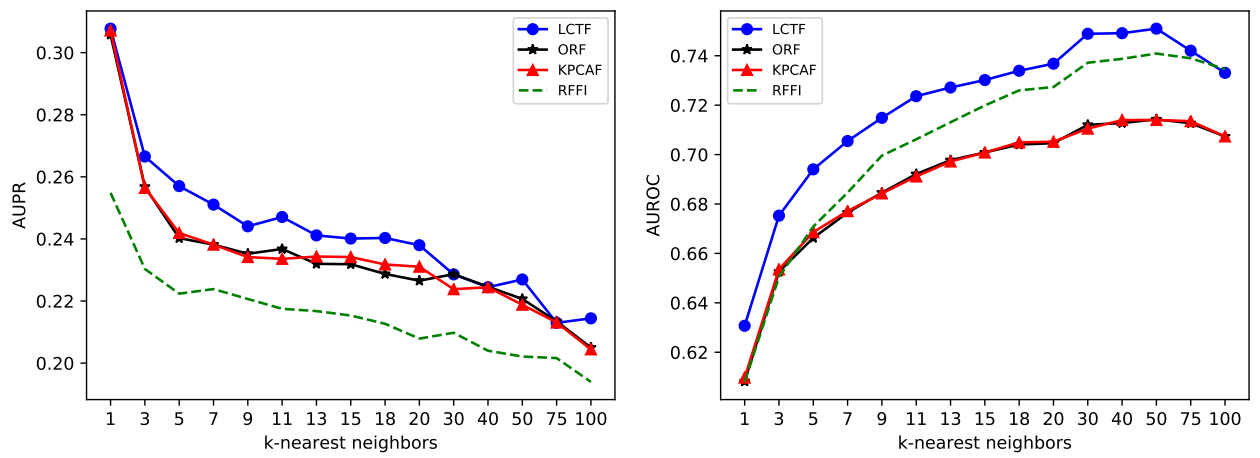

Figure 9: Macro-averaged AUPR and AUROC results for various numbers of nearest neighbors (Medical, MN, PPI, ERN, SRN datasets).

Medical). In order to surpass this impediment, in these datasets, we erased the targets that contain less than 5 positive labels and then computed the macro-averaged AUPR and AUROC scores in the remaining ones, applying a 2 -fold CV. This approach is often used in cases of extreme class imbalanced data [49, 50]. The obtained results are illustrated in Fig. 9. As shown in both Figs. 8 and 9 the results are consistent with the micro-averaged ones, reaffirming the effectiveness of the proposed approach. 


\section{Conclusions}

475 This study aims at highlighting the issue of lacking variance in biological and biomedical data representations and inform the scientific community about it. We also demonstrated the effect on machine learning algorithms. In addition, an efficient feature induction approach was proposed converting the data to a more informative feature representation. The data transformation process is done maximizing the variance between the instances. The generated feature set is compact and therefore computationally efficient. The proposed approach was based on Extremely Randomized Trees inheriting all the advantages of tree ensembles, such as scalability, feature ranking, ability to handle various types of data without the need of preprocessing, and abil-

ity to handle missing values. The efficiency of the method was demonstrated experimentally and the obtained results affirmed its potential.

A possible idea for future work is the employment of different node weighting schemes. An interesting extension could be also the application of deep learning. More specifically, the high dimensional tree induced feature vectors could be converted to low dimensional encodings using auto-encoders.

\section{ACKNOWLEDGMENTS}

The authors acknowledge the KU Leuven research funding.

\section{AUTHOR DISCLOSURE STATEMENT}

No competing financial interests exist. 


\section{References}

[1] I. Yoo, P. Alafaireet, M. Marinov, K. Pena-Hernandez, R. Gopidi, J.-F. Chang, L. Hua, Data mining in healthcare and biomedicine: a survey of the literature, Journal of medical systems 36 (2012) 2431-2448.

[2] A. L. Tarca, V. J. Carey, X.-w. Chen, R. Romero, S. Drăghici, Machine learning and its applications to biology, PLoS Computational Biology 3 (2007) e116.

[3] I. H. Witten, E. Frank, M. A. Hall, C. J. Pal, Data Mining: Practical machine learning tools and techniques, 4th edition, 2016.

[4] D. Kocev, C. Vens, J. Struyf, S. Džeroski, Tree ensembles for predicting structured outputs, Pattern Recognition 46 (2013) 817-833.

[5] G. Tsoumakas, I. Katakis, I. Vlahavas, Mining multi-label data, in: Data mining and knowledge discovery handbook, Springer, US, 2009, pp. 667-685.

[6] M.-L. Zhang, Z.-H. Zhou, A review on multi-label learning algorithms, IEEE Transactions on Knowledge and Data Engineering 26 (2014) 18191837.

[7] L. Breiman, J. Friedman, C. J. Stone, R. A. Olshen, Classification and regression trees, CRC press, 1984.

[8] P. Geurts, A. Irrthum, L. Wehenkel, Supervised learning with decision tree-based methods in computational and systems biology, Molecular Biosystems 5 (2009) 1593-1605. 
[9] L. Breiman, Random forests, Machine learning 45 (2001) 5-32.

[10] E. Spyromitros-Xioufis, G. Tsoumakas, W. Groves, I. Vlahavas, Multitarget regression via input space expansion: treating targets as inputs, Machine Learning 104 (2007) 55-98.

[11] C. J. Burges, A tutorial on support vector machines for pattern recognition, Data mining and knowledge discovery 2 (1998) 121-167.

[12] A. Daemen, B. De Moor, Development of a kernel function for clinical data, in: Engineering in Medicine and Biology Society, 2009. Annual International Conference of the IEEE, pp. 5913-5917.

[13] C. Vens, J. Struyf, L. Schietgat, S. Džeroski, H. Blockeel, Decision trees for hierarchical multi-label classification, Machine Learning 73 (2008) $185-214$.

[14] L. Schietgat, C. Vens, J. Struyf, H. Blockeel, D. Kocev, S. Džeroski, Predicting gene function using hierarchical multi-label decision tree ensembles, BMC bioinformatics 11 (2010) 1.

[15] M. Schrynemackers, L. Wehenkel, M. M. Babu, P. Geurts, Classifying pairs with trees for supervised biological network inference, Molecular BioSystems 11 (2015) 2116-2125.

${ }_{535}$ [16] K. Pliakos, I. Triguero, D. Kocev, C. Vens, Representational power of gene features for function prediction, in: 10th Benelux Bioinformatics Conference (BBC), 2015. 
[17] K. Pliakos, C. Vens, Tree based feature induction for biomedical data, in: 10th international workshop of Machine Learning in Systems Biology (MLSB), The Hague, 3-4 September 2016.

[18] Y. Yamanishi, E. Pauwels, H. Saigo, V. Stoven, Extracting sets of chemical substructures and protein domains governing drug-target interactions, Journal of chemical information and modeling 51 (2011) $1183-1194$.

[19] M.-L. Zhang, Z.-H. Zhou, Ml-knn: A lazy learning approach to multilabel learning, Pattern recognition 40 (2007) 2038-2048.

[20] Y. Saeys, I. Inza, P. Larrañaga, A review of feature selection techniques in bioinformatics, bioinformatics 23 (2007) 2507-2517.

[21] H. Liu, H. Motoda, Feature selection for knowledge discovery and data mining, volume 454, Springer Science \& Business Media, US, 2012.

[22] V. Bolón-Canedo, N. Sánchez-Maroño, A. Alonso-Betanzos, J. M. Benítez, F. Herrera, A review of microarray datasets and applied feature selection methods, Information Sciences 282 (2014) 111-135.

[23] H. Liu, H. Motoda, Feature extraction, construction and selection: A data mining perspective, Springer Science \& Business Media, US, 1998.

[24] M.-L. Zhang, L. Wu, Lift: Multi-label learning with label-specific features, IEEE Transactions on Pattern Analysis and Machine Intelligence 37 (2015) 107-120. 
[25] C. Vens, F. Costa, Random forest based feature induction, in: IEEE 11th International Conference on Data Mining (ICDM), 2011, pp. 744753.

[26] F. Moosmann, E. Nowak, F. Jurie, Fast discriminative visual codebooks using randomized clustering forests., in: Proceedings of the 20th international conference on Neural Information Processing Systems (NIPS), 2006, pp. 985-992.

[27] F. Moosmann, E. Nowak, F. Jurie, Randomized clustering forests for image classification, IEEE Transactions on Pattern Analysis and Machine Intelligence 30 (2008) 1632-1646.

[28] K. Pliakos, C. Vens, Feature induction based on extremely randomized tree paths, in: International workshop of New Frontiers in Mining Complex Patterns, Italy, 2016.

[29] K. Pliakos, C. Vens, Feature induction and network mining with clustering tree ensembles, New Frontiers in Mining Complex Patterns, LNCS 10312 (2017) 3-18.

[30] P. Geurts, D. Ernst, L. Wehenkel, Extremely randomized trees, Machine learning 63 (2006) 3-42.

[31] K. Pliakos, C. Vens, Network representation with clustering tree features, Journal of Intelligent Information Systems (2018).

[32] L. Van Der Maaten, E. Postma, J. Van den Herik, Dimensionality reduction: a comparative, J Mach Learn Res 10 (2009) 66-71. 
[33] L. Cao, K. S. Chua, W. Chong, H. Lee, Q. Gu, A comparison of PCA, KPCA and ICA for dimensionality reduction in support vector machine, Neurocomputing 55 (2003) 321-336.

[34] G. E. Hinton, R. R. Salakhutdinov, Reducing the dimensionality of data with neural networks, Science 313 (2006) 504-507.

[35] H. Blockeel, L. D. Raedt, J. Ramon, Top-down induction of clustering trees, in: Proceedings of the 15th International Conference on Machine Learning, ICML 1998, pp. 55-63.

[36] J. J. Faith, B. Hayete, J. T. Thaden, I. Mogno, J. Wierzbowski, G. Cottarel, S. Kasif, J. J. Collins, T. S. Gardner, Large-scale mapping and validation of Escherichia coli transcriptional regulation from a compendium of expression profiles, PLoS Biology 5 (2007) e8.

[37] K. D. MacIsaac, T. Wang, D. B. Gordon, D. K. Gifford, G. D. Stormo, E. Fraenkel, An improved map of conserved regulatory sites for Saccharomyces cerevisiae., BMC bioinformatics 7 (2006) 113.

[38] Y. Yamanishi, J.-P. Vert, M. Kanehisa, Supervised enzyme network inference from the integration of genomic data and chemical information, Bioinformatics 21 (2005) i468-i477.

[39] C. Von Mering, R. Krause, B. Snel, M. Cornell, S. G. Oliver, S. Fields, P. Bork, Comparative assessment of large-scale data sets of proteinprotein interactions, Nature 417 (2002) 399-403.

[40] A. Elisseeff, J. Weston, A kernel method for multi-labelled classification, 
in: Advances in neural information processing systems, 2002, pp. 681687.

605 [41] J. P. Pestian, C. Brew, P. Matykiewicz, D. J. Hovermale, N. Johnson, K. B. Cohen, W. Duch, A shared task involving multi-label classification of clinical free text, in: Proceedings of the Workshop on BioNLP 2007: Biological, Translational, and Clinical Language Processing, BioNLP 2007, pp. 97-104.

[42] S. Džeroski, D. Demšar, J. Grbović, Predicting chemical parameters of river water quality from bioindicator data, Applied Intelligence 13 (2000) $7-17$.

[43] J. Ruan, W. Zhang, A bi-dimensional regression tree approach to the modeling of gene expression regulation, Bioinformatics 22 (2006) 332340.

[44] F. Pedregosa, G. Varoquaux, A. Gramfort, V. Michel, B. Thirion, O. Grisel, M. Blondel, P. Prettenhofer, R. Weiss, V. Dubourg, J. Vanderplas, A. Passos, D. Cournapeau, M. Brucher, M. Perrot, E. Duchesnay, Scikit-learn: Machine learning in Python, Journal of Machine Learning Research 12 (2011) 2825-2830.

[45] J. D. Hunter, Matplotlib: A 2d graphics environment, Computing In Science \& Engineering 9 (2007) 90-95.

[46] A. Hyvärinen, E. Oja, Independent component analysis: algorithms and applications, Neural networks 13 (2000) 411-430. 
${ }_{625}$ [47] S. T. Roweis, L. K. Saul, Nonlinear dimensionality reduction by locally linear embedding, science 290 (2000) 2323-2326.

[48] J. B. Tenenbaum, V. De Silva, J. C. Langford, A global geometric framework for nonlinear dimensionality reduction, science 290 (2000) 2319-2323.

[49] M.-L. Zhang, Y.-K. Li, X.-Y. Liu, Towards class-imbalance aware multilabel learning., in: International Joint Conference on Artificial Intelligence (IJCAI), 2015, pp. 4041-4047.

[50] Z. A. Daniels, D. N. Metaxas, Addressing imbalance in multi-label classification using structured hellinger forests., in: AAAI, 2017, pp. $1826-1832$. 